subtype), type of medication during pregnancy and immediate postpartum changes aOR $0.3395 \% \mathrm{CI}[0.03 ; 3.40], \mathrm{p}=0.35$.

Conclusion. A high number of women with bipolar disorder are taking medication before delivery and in the majority, antipsychotics are prescribed. The postnatal recurrence rate in both medicated and unmedicated women is high. These results are in line with existing literature. Further work is needed in larger samples to provide clinical guidance for women and their clinicians.

\section{Psilocybin: the magic medicine for depression?}

Amber Elyse Corrigan*, Ella Burchill, Lucy Pelton, Alessia Marrocu, Adele Mazzoleni and Lydia Shackshaft

King's College London

${ }^{*}$ Corresponding author.

doi: 10.1192/bjo.2021.456

Aims. Depression is the single largest contributor to global disability. However, effective treatments are currently lacking, resulting in a significant burden of treatment-resistant depression (TRD). Psilocybin, a serotonergic psychedelic, found as the active compound in 'magic mushrooms', has been proposed as a novel therapeutic avenue for TRD. We aimed to evaluate the future feasibility and implications of psilocybin as a new antidepressant therapy.

Method. We reviewed and critically analysed the available literature on the efficacy and safety of psilocybin as a treatment for depression, and the potential pharmacological and psychological mechanisms of the therapeutic benefit. We discussed the relative contributions to this therapeutic effect of the pharmacological drug treatment, placebo effects, and the context and parameters of the psychotherapeutic experience. We reviewed legal, social, and economic barriers to primary research and clinical implementation.

Result. Psilocybin in combination with psychotherapy has been shown to be safe and effective in TRD. Its mechanism of action in TRD has not been fully elucidated, however reviewing functional neuroimaging studies demonstrated disparate short and long-term modifications of default mode network connectivity, suggested to represent a 'reset' mechanism of acute modular disintegration and subsequent reintegration which restores normal function, reviving emotional responsiveness.

Research suggests psychedelic treatment induces lasting personality, belief and attitude changes. The psychedelic drug itself, the context of the psychotherapeutic experience, and the postdrug integration therapy all appear to have a significant role. Preparation prior to treatment, the environment, context and support during the psychedelic experience itself, and the following long-term integration and support process must be considered.

Despite novel findings Psilocybin is a Schedule I drug; this imposes a persisting ethical barrier to clinical use. Prohibition of psilocybin results in high costs of drug supply, and potential for harmful drug-seeking behaviours. Therefore, complex sociopolitical factors currently limit wider implementation.

Conclusion. Psilocybin in combination with psychotherapy is safe and effective in TRD. The interacting and elusive therapeutic mechanisms have implications for clinical implementation. Preparation prior to treatment, the physical and social environment in which the psychedelic experience takes place, and longterm integration and support are considered to play a significant role. Optimisation of these parameters and cost-benefit analyses are required prior to this being feasible as a widely available therapy. Systemic legislative, political and social change will also be key to enable widespread clinical use. The promise of this therapy on a background of inadequate current antidepressant treatments indicates these must be a priority.
Buspirone in obsessive-compulsive disorder: a potential dark horse?

Kabir Garg ${ }^{1 \star}$ and Himanshu Tyagi ${ }^{2}$

${ }^{1}$ Oxleas NHS Foundation Trust and ${ }^{2}$ UCL Queen Square Institute of Neurology, The National Hospital for Neurology and Neurosurgery ${ }^{\star}$ Corresponding author.

doi: 10.1192/bjo.2021.457

Aims. Pharmacological management of Obsessive-Compulsive Disorder (OCD) presents a challenge in modern psychiatry. While most patients respond preferably to serotonin re-uptake inhibitors (SRI), the response is usually delayed by several weeks leading to an insufficient short term management of anxiety. It is also frequently inadequate and needs higher doses and augmentation in many instances. Investigating newer pharmacological strategies to address such treatment gaps has always been of interest. Buspirone is a novel anxiolytic medication with additional weak antidepressant and poor anti-psychotic effects. It is the only medication in its category, i.e. azapriones. It has comparable anti-anxiety efficacy to that of benzodiazepines without their sedating or habit forming effects, and has been demonstrated to moderate serotonin and other monoamine neurotransmission with a favourable safety profile.

Method. We reviewed the literature pertaining to the use of Buspirone in OCD for both as a primary anti-obsessive agent and for a potential secondary role in management of chronic anxiety and/or anxiety disorders comorbid to OCD.

Result. The results of a number of case reports and open trials have been positive while controlled trials have shown contradictory results. In a double blind RCT comparing clomipramine and buspirone, significant improvement was found in both groups with no differences between the two. Further two trials observing buspirone augmentation of clomipramine and fluoxetine treatment respectively, in a double-blind placebo controlled design reported significant improvement in the treatment as opposed to the placebo arm. Another double-blind placebo controlled study of buspirone augmentation of fluvoxamine resistant patients did not show significant benefits as an anti-obsessional agent, but notable anxiolytic effects were reported. In all the trials buspirone was largely well tolerated and did not pose any significant interactions with other psychotropic agents or dependence potential. Conclusion. Buspirone is a pharmacologically unique agent with a good safety profile. Given the robust anxiolytic effects of this Peron along with complex neurotransmission modulatory effects coupled with a favourable tolerance and dependents profile might make buspirone an attractive novel pharmacological agent for augmentation in OCD . Further controlled studies to better establish effectiveness and deciphering if certain patients may respond to its use over others, are warranted

\section{The links between the amount of antipsychotic medication prescribed at GP practice level, local demographic factors and medication selection}

Adrian Heald ${ }^{1 \star}$, Mike Stedman ${ }^{2}$, Sanam Farman ${ }^{3}$, Mark Davies $^{4}$, Roger Gadsby ${ }^{5}$ and David Taylor ${ }^{6}$

${ }^{1}$ University of Manchester, Salford Royal Hospital; ${ }^{2}$ Res Consortium; Chaw Khine, Kings Mill Hospital; ${ }^{3}$ Mersey Deanery Psychiatry Rotation; ${ }^{4}$ Res Consortium; ${ }^{5}$ University of Warwick and ${ }^{6}$ South London and Maudsley NHS Foundation Trust

${ }^{\star}$ Corresponding author.

doi: 10.1192/bjo.2021.458 
Aims. To examine the factors that relate to antipsychotic prescribing in general practices across England and how these relate to cost changes in recent years.

Background. Antipsychotic medications are the first-line pharmacological intervention for severe mental illnesses(SMI) such as schizophrenia and other psychoses, while also being used to relieve distress and treat neuropsychiatric symptoms in dementia.

Since 2014 many antipsychotic agents have moved to generic provision. In 2017_18 supplies of certain generic agents were affected by substantial price increases.

Method. The study examined over time the prescribing volume and prices paid for antipsychotic medication by agent in primary care and considered if price change affected agent selection by prescribers.

The NHS in England/Wales publishes each month the prescribing in general practice by BNF code. This was aggregated for the year 2018_19 using Defined Daily doses (DDD) as published by the World Health Organisation Annual Therapeutic Classification (WHO/ATC) and analysed by delivery method and dose level. Cost of each agent year-on-year was determined.

Monthly prescribing in primary care was consolidated over 5 years (2013-2018) and DDD amount from WHO/ATC for each agent was used to convert the amount to total $\mathrm{DDD} /$ practice.

Result. Description

In 2018_19 there were $10,360,865$ prescriptions containing 136 million DDD with costs of $£ 110$ million at an average cost of $£ 0.81 / \mathrm{DDD}$ issued in primary care. We included 5,750 GP Practices with practice population $>3000$ and with $>30$ people on their SMI register.

Effect of price

In 2017_18 there was a sharp increase in overall prices and they had not reduced to expected levels by the end of the 2018_19 evaluation year. There was a gradual increase in antipsychotic prescribing over 2013-2019 which was not perturbed by the increase in drug price in $2017 / 18$.

Regression

Demographic factors

The strongest positive relation to increased prescribing of antipsychotics came from higher social disadvantage, higher population density(urban), and comorbidities e.g. chronic obstructive pulmonary disease(COPD). Higher \%younger and \%older populations, northerliness and non-white (Black and Minority Ethnic (BME)) ethnicity were all independently associated with less antipsychotic prescribing.

Prescribing Factors

Higher DDD/general practice population was linked with higher \%injectable, higher \%liquid, higher doses/prescription and higher \%zuclopenthixol. Less DDD/population was linked with general practices using higher \%risperidone and higher spending/dose of antipsychotic.

Conclusion. Higher levels of antipsychotic prescribing are driven by social factors/comorbidities. The link with depot medication prescriptions, alludes to the way that antipsychotics can induce receptor supersensitivity with consequent dose escalation.

\section{Different onset patterns of monthly paliperidone palmitate in hospitalised patient diagnosed with schizophrenia}

Javier Herrera-Sánchez ${ }^{*}$, Julia Aznar, Leire Izaguirre and Santiago Ovejero

Hospital Universitario Fundación Jiménez Díaz

${ }^{\star}$ Corresponding author.

doi: 10.1192/bjo.2021.459
Aims. Paliperidone palmitate long-acting injectable (PPLAI) initially requires two loading doses of 150 and $100 \mathrm{mg}$ on days 1 and 8 ( \pm 4 days) intramuscularly. In clinical practice, different PPLAI initiation patterns have been observed. The aim of this study is to describe different PPLAI onset patterns in hospitalised patients diagnosed with schizophrenia.

Method. A naturalistic, transversal, retrospective, descriptive study was carried out. Patients were recruited in the adult inpatient unit of Hospital Universitario Jiménez Díaz (Madrid, Spain) from November 2012 to February 2021. During this period, a total of 357 patients were treated with PPLAI, 172 of them were diagnosed with schizophrenia and, among these, 24 received an atypical onset pattern during hospitalization. Different PPLAI onset patterns, PPLAI dose at discharge and number of days hospitalised were analysed. This study followed the Declaration of Helsinki principles and was approved by the Local Ethics Committee. All participants gave written informed consent.

Result. The sample presents 24 patients (17 men, 7 women) that represents $6.72 \%$ of a global sample, with an average age of 40.21 years (men 35.59 years vs. women 51.43 years). In this study, different PPLAI onset patterns were described: those receiving $150-150 \mathrm{mg}$ represent $25 \%$ of the sample $(n=6)$, as do those receiving $100-75 \mathrm{mg}$, also representing $25 \%$ of the sample $(n=6)$. The rest of the onset patterns were: $150-75 \mathrm{mg}(20.83 \%$, $\mathrm{n}=5), \quad 100-100 \mathrm{mg}(12.5 \%, \mathrm{n}=3), 150-75 \mathrm{mg} \quad(4.16 \%, \mathrm{n}=1)$, $100-50 \mathrm{mg}(4.16 \%, \mathrm{n}=1), 75-100 \mathrm{mg}(4.16 \%, \mathrm{n}=1)$, and $75-75$ $\mathrm{mg}(4.16 \%, \mathrm{n}=1)$. The average hospital stay is 17.88 days. The PPLAI maintenance dose at discharge was $104.17 \mathrm{mg} / \mathrm{month}$. The group of patients who received two doses of $150 \mathrm{mg}$ (150-150 mg) had an average length of stay of 27.67 days compared to the rest of the patients who had an average length of stay of 15.12 days, this difference being statistically significant $(\mathrm{p}=0.010)$. The $150-150 \mathrm{mg}$ group was discharged with a mean maintenance dose of $141.67 \mathrm{mg}$ versus the other patients who needed a mean maintenance dose of $91.18 \mathrm{mg}$, which was also statistically significant $(\mathrm{p}=0.001)$.

Conclusion. The most used pattern of atypical onset of PPLAI in this sample is $150-150 \mathrm{mg}$ and $100-75 \mathrm{mg}$. Patients treated with 150-150 mg loading pattern are hospitalized for a longer period and needed higher maintenance dose at discharge. Further studies are needed.

\section{Adjunct pharmacotherapy for psychotherapy}

Jessica Lochtenberg ${ }^{1 \star}$, Ari Kirshenbaum ${ }^{2}$ and Matthew Johnson ${ }^{3}$ ${ }^{1}$ Royal College of Surgeons in Ireland; ${ }^{2}$ Saint Michael's College and ${ }^{3}$ Johns Hopkins University

${ }^{*}$ Corresponding author.

doi: $10.1192 /$ bjo.2021.460

Aims. A variety of pharmacotherapies have been used to assist the psychotherapy process as "adjunctive therapies." These drugs are used in an acute, targeted fashion, such that they are explicitly delivered in the context of psychotherapy for anxiety, mood and substance-dependence disorders (SUDs). Our narrative review highlights the potential of medically-assisted psychotherapy by outlining the current state of research on few of these medications and describing the basic science that supports their use.

Method. Firstly, we researched an assortment of medications that have been used off-label to enhance psychotherapy, and selected a few that have received the most empirical attention in preclinical and clinical-trial settings. Our review of clinical trials focused on three of the most common psychiatric ailments. For all studies reviewed, we identify the strengths and weaknesses of the data 